\title{
High Helmholtz Sound Prediction Generated by Confined Flows and Propagation within Ducts
}

\author{
Morteza Bayati and Mehran Tadjfar \\ Aerospace Engineering Department, Amirkabir University of Technology, Tehran 15875-4413, Iran
}

(Received 1 January 2013; revised 26 October 2013; accepted 5 March 2014)

\begin{abstract}
A hybrid method for the computation of noise radiation by a confined flow is used in this study. The proposed approach is appropriate and quite powerful for high Helmholtz numbers (i.e. when the turbulence/body interaction region is acoustically non-compact). The validation of this method is checked by comparing it with the analytical results of the tailored Green's function to the spinning of two vortex filaments in an infinite 2-D duct. The method is applied to the prediction of sound in a duct obstructed by a diaphragm. The sound sources generated by the fluctuations in the flow field are computed by means of an incompressible Large Eddy Simulation (LES). These sources are fed into a 2-D acoustic propagation Boundary Element Model (BEM). The predicted total acoustic power is $1.8 \mathrm{~dB}$ higher than the result obtained in the literature by Direct Numerical Calculation (DNC) and extrapolated experimental data for the same pipe configuration.
\end{abstract}

\section{NOMENCLATURE}

$\begin{array}{ll}c_{0} & \text { Sound speed } \\ \phi & \text { Field variable } \\ ()_{0} & \text { Reference quantities } \\ u_{i} & \text { Velocity components } \\ ()_{L} & \text { Flow quantity } \\ ()^{\prime} & \text { Fluctuation } \\ ()_{a} & \text { Acoustic quantity } \\ n_{i} & \text { Normal vector } \\ \delta_{i j} & \text { Kronecker delta } \\ T_{i j} & \text { Lighthill tensor } \\ H_{0}^{1} & \text { Hankel function of the first kind } \\ C(x) & \text { Solid angle } \\ x & \text { Source position (vector } \vec{x}) \\ y & \text { Listener position (vector } \vec{y}) \\ p & \text { Pressure } \\ V_{\varepsilon} & \text { Exclusion volume } \\ f & \text { Frequency } \\ \delta_{e} & \text { Momentum thickness } \\ I & \text { Acoustic intensity } \\ P & \text { Acoustic power } \\ \omega & \text { Angular frequency }(\omega=2 \pi f) \\ G & \text { Green function }\end{array}$

\section{INTRODUCTION}

In many practical applications, sound is generated by the interaction of turbulent flow with solid walls. In this situation, the sound wave experiences multiple reflections before propagation to a far field. Therefore, the sound spectrum exhibits rich frequencies content consisting of broadband and tonal components. To predict the acoustics field in these situations, a general aero-acoustic framework is required. More importantly, the employed method must often avoid many simplifying assumptions about geometry, compactness, or frequency content of sound sources.

The prediction of flow-induced noise requires accounting for the physics of both unsteady flow and the sound wave, simultaneously, since both are a solution of the compressible Navier-Stokes equation. The basic difficulties for such computations are numerous disparities between energies and lengthscale in the turbulent flow and the sound field. Sound waves carry only a minuscule fraction of the flow energy, and a highorder numerical scheme is required to keep the sound wave intact. These fundamental differences are exacerbated in a low Mach number flow, ${ }^{1}$ where the radiated acoustic power is smaller than the hydrodynamic flow power by roughly $O\left(M^{4}\right)$. In addition, the acoustic CFL number imposes extremely small time steps on the numerical solution in order to resolve both acoustics and hydrodynamics. That is why it is commonly accepted that hybrid methods are more appropriated for low Mach number flows. An example of a two-step or hybrid method for nearly incompressible flows is Lighthill, ${ }^{2}$ who formally separated acoustics from hydrodynamics by introducing his acoustic analogy. It was shown that the flow mechanism that produced noise could be expressed in the form of equivalent sources in a uniform medium at rest, chosen as a representation of the propagation region surrounding the listener. The idea of equivalent sources has proven to be quite powerful at low Mach numbers. Curle ${ }^{3}$ extended the Lighthill analogy to predict the sound of turbulent-body interaction by introducing a dipole source. Ffowcs Williams and Hawkings ${ }^{4}$ generalized previous analogies to account for moving sources and the resulting Doppler effects.

Most of the works in literature are exterior problems. In the present work, we are concerned with the noise generated by confined flows and its propagation within the ducting. The interaction of pipe flow with singularities like diaphragm, valve contractions, or pipe junctions are sources of internal noise in industrial duct networks. ${ }^{5,6}$ The problem of aerodynamics sound generation in pipes, allowing the aeroacoustics analogy by Davies and Ffowcs Williams, ${ }^{7}$ showed that the acoustic efficiency of turbulence within a straight infinite duct varies with frequency from a dipole-like behaviour below the cut-off fre- 